\title{
Lorentz Violating Julia-Toulouse Mechanism
}

\author{
Patricio Gaete * $^{2}$ \\ Departamento de Física, Universidad Técnica F. Santa María, Valparaíso, Chile \\ Clovis Wotzasel $\oplus$ \\ Instituto de Física, Universidade Federal do Rio de Janeiro, Brazil
}

(Dated: May 29, 2018)

\begin{abstract}
We propose a new Lorentz invariant violating extension for the pure photonic sector of the Standard Model due to the condensation of topological defects in the context of the Julia-Toulouse mechanism. Possible physical consequences leading to direct measurable effects over the confining properties of the elementary particles are explored.

PACS numbers: 11.10.Lm, 11.15.-q and 11.30.Pb
\end{abstract}

This work is devoted to study both the condensation of topological defects(TD) 1] displaying Lorentz symmetry violation (LIV) 2] and the electromagnetic properties of extended charged objects living inside the condensate 3. It is shown that LIV-condensate may result from a (modified) Julia-Toulouse mechanism (JTM) that considers a non-homogeneous nucleation of TD. The condensate characterizes the new vacuum of the theory now displaying LIV as a spontaneous symmetry breaking (SSB) process with striking consequences over the diferent phases of the electromagnetic sector of an extended standard model. This breaking of Lorentz symmetry (LS) can be parametrized by constant tensor fields introducing the notion of a preferred frame - the rest frame of the condensate [4]. This mechanism of LIV introduces the notion of effective-brane displaying a jumping of dimension mechanism and allowing for the extended charges to couple to tensor fields of lower ranks they normally would in the presence of the Lorentz symmetry.

Lorentz invariance is one of the cornerstones of modern quantum field theory (QFT). This seems to be an exact symmetry respected by the Standard Model (SM) of elementary particles [5]. This theory has local LS and has been confirmed to an extremely high precision. The observation of LIV would therefore give a positive signature for the existence of a new and unconventional physics. The necessity of a new scenario has been suggested both to answer theoretical difficulties in quantum gravity [6, 7] and to the exciting new possibility of high-precision experimental tests searching for small violations of the LS [8]. However, because LS is so deeply-rooted into the structure of QFT, it is difficult to design a theory without a radical revision on its foundations supporting those numerous experiments searching for small LS violations arising as the low energy limit of such yet unknown ultimate theory valid at the Planck scale [9].

Since our low-energy theories are relativistic QFT, it is interesting to explore possible new phenomena in this

\footnotetext{
*Electronic address: patricio.gaete@usm.cl
}

†Electronic address: clovis@if.ufrj.br framework that could produce departures from exact Lorentz invariance. Among these tentatives, probably the most studied framework is the standard model extension (SME), consisting of the minimal SM plus small Lorentz (and CPT) violating terms which has provided a theoretical background for many experiments of Lorentz and CPT violations such as: neutrino and neutralmeson oscillations, clock-comparison tests on Earth and in space, the motion of a spin-polarized torsion pendulum, hydrogen and antihydrogen spectroscopy, QED in Penning traps, cosmological birefringence, microwave cavities, baryon asymmetry, muon properties, etc 10.

The basic idea in the SME is that the theory transforms normally under rotations and boosts of the observer's inertial frame but apparent Lorentz violations could appear when the particle fields are rotated or boosted relative to the vacuum tensor expectation values. This is called particle Lorentz transformations. One interesting possibility is the SSB of LS in an ultimate fundamental theory. Basically the interactions among tensor fields trigger the formation of nonzero vacuum expectation values for Lorentz tensors. The presence of these background quantities throughout spacetime implies that Lorentz symmetry is spontaneously broken.

The usual SM lacks the necessary dynamics to break LS spontaneously, since the interactions triggering the Lorentz SSB would destabile the empty vacuum. Spontaneous breaking could however occur in more complicated theories involving extended objects called p-branes known to have dynamics of the necessary type. For most situations at energies well below the scale of the underlying theory, it suffices to study the subset of the full Standard-Model Extension for which the gauge structure of the usual Standard Model are changed. Particularly interesting examples are the LIV electrodynamics, 11] and [12], including either even or odd CPT-violating terms or both. These modified electrodynamics that maintain the usual gauge invariance and are covariant under observer Lorentz transformations read

$$
\begin{aligned}
\mathcal{L}_{\text {photon }}= & -\frac{1}{4} F_{\mu \nu} F^{\mu \nu}+\frac{\alpha}{2}(b)^{\kappa} \epsilon_{\kappa \lambda \mu \nu} A^{\lambda} F^{\mu \nu} \\
& -\frac{\beta}{4}(M)_{\kappa \lambda \mu \nu} F^{\kappa \lambda} F^{\mu \nu}
\end{aligned}
$$


In [1] a Maxwell-Chern-Simons like model $(\beta=0)$ was studied to discuss relevant cosmological data while in 12 both odd and even CPT-violating terms were included but emphasis was given to the $\alpha=0$ case. These theories have predicted several peculiar features that led to sensitive tests of LIV.

The importance of the phenomenological model proposed here resides on the fact that it provides us with an extension that comes from a well-known physical mechanism very much associated with the dual superconductivity. In this paper an extension for the electromagnetic sector that breaks LS being parametrized by a constante vector $v_{\mu}$ is proposed to control the dynamics of the electromagnetic fields in the presence of LIV, considering the vacuum as a Lorentz violating condensate.

We are then led to investigate possible mechanism such that a reasonable quantum field theory might spontaneously break LI. Taking inspiration from theoretical work on superconductivity as the result of condensation of TD (dual Meisner effect) we argue for the existence of theories with Lorentz invariant bare Lagrangians where the formation of a condensate of TD, being energetically favorable, leads to a non-Lorentz invariant vacuum characterized by a constant vector induced by the condensation. The new vacuum will display electric or magnetic properties depending on the spacetime nature of this induced vector. The final effective theory for the electromagnetic fields in this LIV-vacuum reads,

$$
\mathcal{L}_{\text {eff }}=-\frac{1}{4} F_{\mu \nu} g^{\nu \beta}\left[g^{\mu \alpha}+g^{2} \frac{v^{\mu} v^{\alpha}}{\Delta+m^{2}}\right] F_{\alpha \beta}
$$

This model is an effective theory derived using an extension of the JTM in the presence of broken symmetries.

The JTM is a condensation process dual to the Higgs mechanism, proposed by Quevedo and Trugenberger (QT) 1]. It is intended to describe phenomenologically the electromagnetic behavior of anti-symmetric tensors in the presence of of magnetic-branes (topological defects) that eventually condense due to thermal and quantum fluctuations. Defects are classical solutions of the equations of motion and may be classified as topological or nontopological. Topological defects appear in models that support spontaneous symmetry breaking. They are important also in Cosmology and Condensed Matter Physics. In their derivation of this mechanism, QT demanded a few features to take into consideration the contributions of the fluctuations of the TD. However, they did not worry about the exact (microscopic) description of the condensation process. As so, the determination of the critical values for the coupling constant or the density of the condensate do not become an issue. Instead, the focus was in the phenomenological description of the electromagnetic behavior after brane condensation phenomenom had taken place. We shall follow a similar atitude here. To study this nucleation process the strategy is to look for a gauge invariant action, linear, with up to two derivative dynamics that should recover the normal vacuum in the limit of the dilute phase. Finally, among those features, Lorentz symmetry was included before while here we shall relax this last demand. Next, the consequences of this LIV extension are examined. The LIV model studied here is obtained following a previous work of the authors using the JTM $[3]$ while the analytical work follows [13, 14].

In the JTM the topological defects play the role of magnetic objects. They are characterized by their intensity, described by the coupling constant $g$, and the Chern-Kernel $\Lambda_{q+1}$, to be detailed below, describes their space dimension $p$ and localization. In [1] it was found that, starting from a Maxwell-like theory longitudinally coupled to p-branes, the JTM produces a model for free massive condensate:

$$
\int\left[F_{p+1}\left(A_{p}\right)-g \Lambda_{p+1}\right]^{2} \rightarrow \int\left[F_{p+2}^{2}\left(\Lambda_{p+1}\right)-m^{2} \Lambda_{p+1}^{2}\right],
$$

where $m$ is a free phenomenological mass parameter. Notice that in the JTM, the Chern-Kernel $\Lambda_{p+1}$ is elevated to the status of a propagating massive field, describing the low-energy (long wavelength) hydrodynamical modes of the newly formed condensate. The JTM is dual to the well known Higgs mechanism (HM) where the gauge potential "eats up" the degree of freedom of the condensate. There is however a striking difference - there is a rankjump here that is absent in the HM since $A_{p} \rightarrow \Lambda_{p+1}$. In the JTM, on the other hand, by absorbing the degrees of freedom of the original massless tensor $A_{p}$, it is the condensate field that becomes massive. In this way the mass density of the condensate becomes a free parameter to be determined by the experiment. QT have shown that in the dilute phase limit $(m \rightarrow 0)$ one indeed recovers the original theory. In summary, the JTM provides very successfuly phenomenological description for the magnetic condensate through the effective Chern-Kernel field dynamics.

In [3] the present authors have used the formalism proposed in [1] to study the interplay between screening and confinement due to the condensation of topological defects and try to fix the value of the free parameter $m$ by connecting it to some observable of the theory: the measurement of the confining effective potential of a pair of very massive test charges. Using the QT phenomenology we were able to discuss the dynamics of the extended charges ( $p$-branes) inside the new vacuum provided by the condensate. The model proposed in [3] considered the TD coupled both longitudinally and transversally to two different tensor potentials, $A_{p}$ and $B_{q}$, such that $p+q+2=D$ which, before condensation reads, [17]

$$
\begin{aligned}
\mathcal{L}_{d} & \propto\left[F_{p+1}\left(A_{p}\right)-g \Lambda_{p+1}\right]^{2} \\
& +g B_{q} \epsilon^{q ; 1 ; p+1} \partial_{1} \Lambda_{p+1}+H_{q+1}^{2}\left(B_{q}\right),
\end{aligned}
$$

where the current $J^{q}=\epsilon^{q ; 1 ; p+1} \partial_{1} \Lambda_{p+1}$, represents the world-volume of a $(q-1)$-brane. After condensation one obtains

$$
\begin{aligned}
\mathcal{L}_{c} & \propto H_{q+1}^{2}\left(B_{q}\right)+g B_{q} \epsilon^{q ; 1 ; p+1} \partial_{1} \Lambda_{p+1} \\
& +F_{p+2}^{2}\left(\Lambda_{p+1}\right)-m^{2} \Lambda_{p+1}^{2}
\end{aligned}
$$


showing an interacting theory displaying a $B \wedge F$-type of coupling between the $B_{q}$ potential with the tensor $\Lambda_{p+1}$ carrying the degrees of fredom of the condensate. This theory displays a second mechanism of mass generation. It is exactly the interplay between these two mass parameters that controls the screening/confining character of the theory.

This is our starting point in order to study the nature of the LIV-vacuum of the anti-symmetric tensor electromagnetic-like theory. In [3] the effective theory that results from integrating out the fields representing the vacuum-condensate, describing the dynamics of the quanta exchanged by the test charges when immersed into the condensate, was given as

$$
\mathcal{L}_{e f f} \propto F_{q+1}\left(B_{q}\right)\left[1+\frac{g^{2}}{\Delta+m^{2}}\right] F^{q+1}\left(B_{q}\right) .
$$

In this work we want to explore the possibility that an analogous process occurs as the result of an spontaneous breaking of the Lorentz symmetry. In their work, QT assumed a homogeneous nucleation of the TD. We want to disccuss now what would the outcome of condensation be in the presence of symmetry violation. What is instrumental for the development of SSB-JTM is the notion of "effective brane". In the presence of a constant rank- $m$ tensor, say $T_{m}$, a charge of dimension $(q+m-1)$ will appear as an effective brane of dimension $(q-1)$, $\Lambda_{p+1-m} \rightarrow \tilde{\Lambda}_{p+1}=T_{[m} \Lambda_{p-m+1]}$. This lower-dimensional brane represents the intersection of the subspace transverse to the $T_{m}$ tensor with the world-volume of the $(q+m-1)$-brane giving the world-volume for the effective $(q-1)$-brane. Then, for instance, for a constant vector, $T_{1} \equiv v_{1}$, the world-volume of a membrane is changed to an effective string, that now couples to Kalb-Ramond potentials and a string behaves as if it were a particle, coupling to vector fields and so on. The obvious consequence of this mechanism is that higher-dimensional objects are now able to couple to lower-ranked potentials. Therefore, upon nucleation, this dimension-changing mechanism for the branes becomes a rank-changing mechanism for the condensates. This rank-changing mechanism alters the JTM that now has a condensed action given as

$$
\begin{aligned}
\mathcal{L}_{S S B} & \propto H_{q+1}^{2}\left(B_{q}\right)+g T_{[l} B_{q]} \epsilon^{q ; 1 ; p+1} \partial_{1} \Lambda_{p-l+1} \\
& +F_{p-l+2}^{2}\left(\Lambda_{p-l+1}\right)+m^{2} \Lambda_{p-l+1}^{2}
\end{aligned}
$$

For a condensate, where LIV is characterized by a constant vector $v_{1} \equiv v_{\mu}$ we obtain,

$$
\begin{aligned}
\mathcal{L}_{L I V} & =H_{q+1}^{2}\left(B_{q}\right)+g v_{[1} B_{q]} \epsilon^{q+1 ; 1 ; p} \partial_{1} \Lambda_{p} \\
& +F_{p+1}^{2}\left(\Lambda_{p}\right)+m^{2} \Lambda_{p}^{2} .
\end{aligned}
$$

Upon integration of the field charaterising the LIVcondensate we obtain the result expressed before in (2) Next we shall study the consequences of this mechanism over the confining and screening phases of the charges living inside this condensate. To this end we shall focus on
$D=3$ space-time models that might be of revelevance in condensed matter physics. We now examine the interaction energy between static pointlike sources for the model under consideration, along the lines of Refs. 13]. This can be done by computing the expectation value of the energy operator $H$ in the physical state $|\Phi\rangle$ describing the sources, which we will denote by $\langle H\rangle_{\Phi}$.

The starting point is the 3D JTM Lagrangian density:

$$
\mathcal{L}=-\frac{1}{4} F_{\mu \nu}^{2}+\frac{g}{2} \phi \varepsilon^{\mu \nu \lambda} v_{\mu} F_{\nu \lambda}+\frac{1}{2} \partial_{\mu} \phi \partial^{\mu} \phi-\frac{m^{2}}{2} \phi^{2},
$$

where $m$ is the mass for the condensate field $\phi$. We restrict ourselves to static scalar fields, a consequence of this is that one may replace $\Delta \phi=-\nabla^{2} \phi$, with $\Delta \equiv \partial_{\mu} \partial^{\mu}$. It also implies that, after performing the integration over $\phi$, the effective Lagrangian density is given by

$$
\mathcal{L}_{e f f}=-\frac{1}{4} F_{\mu \nu}^{2}-\frac{g^{2}}{8} V^{\nu \lambda} F_{\nu \lambda} \frac{1}{\nabla^{2}-m^{2}} V^{\gamma \beta} F_{\gamma \beta}+A_{\mu} J^{\mu},
$$

where $V^{\nu \lambda} \equiv \varepsilon^{\mu \nu \lambda} v_{\mu}$ and $J^{\mu}$ is the external current of the test charges and $(\mu, \nu=0,1,2)$ and $(i, k=1,2)$. It should be clear that the theory (10) depends on the spacetime character of the constant vector $v_{\mu}$. We shall consider different possibilities next.

Spacelike background case. To obtain the interaction energy in the $V^{0 i} \neq 0$ and $V^{i j}=0$ case, we compute the Hamiltonian density of (10)

$$
\mathcal{H}=\left\{\frac{1}{2} \Pi^{2}+\frac{g^{2}}{2} \frac{(\mathbf{V} \cdot \boldsymbol{\Pi})^{2}}{\left(\nabla^{2}-M^{2}\right)}+\frac{1}{2} B^{2}+c(x) \Gamma(x)\right\},
$$

where $\Gamma_{1}(x) \equiv \partial_{i} \Pi^{i}-J^{0} \approx 0$ is the Gauss law, $M^{2} \equiv$ $m^{2}+g^{2} V^{2}$ and $B$ is the magnetic field. To fix gauge symmetry we adopt the linear gauge discussed in our previous works $[3,14$. Next the physical state is constructed, following Dirac 15,

$$
\begin{aligned}
& |\Phi\rangle \equiv\left|\bar{\Psi}(\mathbf{y}) \Psi\left(\mathbf{y}^{\prime}\right)\right\rangle \\
& =\bar{\psi}(\mathbf{y}) \exp \left(i e \int_{\mathbf{y}^{\prime}}^{\mathbf{y}} d z^{i} A_{i}(z)\right) \psi\left(\mathbf{y}^{\prime}\right)|0\rangle
\end{aligned}
$$

where $|0\rangle$ is the physical vacuum state and the line integral appearing in the above expression is along a spacelike path starting at $\mathbf{y}^{\prime}$ and ending at $\mathbf{y}$, on a fixed time slice.

We now turn to the problem of obtaining the interaction energy between pointlike sources in the model under consideration. A fermion is localized at $\mathbf{y}^{\prime}$ and an antifermion at $\mathbf{y}$. One might show that the interaction energy is

$$
\langle H\rangle_{\Phi}=\left\langle\Phi\left|\int d^{2} x\left\{\frac{1}{2} \boldsymbol{\Pi}^{2}+\frac{g^{2}}{2} \frac{(\mathbf{V} \cdot \boldsymbol{\Pi})^{2}}{\left(\nabla^{2}-M^{2}\right)}+\frac{1}{2} B^{2}\right\}\right| \Phi\right\rangle
$$

where $\langle H\rangle_{\Phi}=\langle H\rangle_{0}+V^{(1)}+V^{(2)}$ with $\langle H\rangle_{0}=\langle 0|H| 0\rangle$ giving the background vacuum term while the contributions of the test charges are given by $V^{(1)}$ and $V^{(2)}$ and 
read,

$$
V^{(1)}=-\frac{e^{2}}{2 \pi} K_{0}\left(M\left|\mathbf{y}-\mathbf{y}^{\prime}\right|\right)
$$

and

$$
V^{(2)}=\frac{e^{2} m^{2}}{4 M}\left|\mathbf{y}-\mathbf{y}^{\prime}\right|
$$

By putting together Eqs.(14) and (15), we obtain for the total interaction energy $\left(L \equiv\left|\mathbf{y}-\mathbf{y}^{\prime}\right|\right)$

$$
V(L)=-\frac{e^{2}}{2 \pi} K_{0}(M L)+\frac{e^{2} m^{2}}{4 M} L .
$$

Timelike background case. Now we focus on the case $V^{0 i}=0$ and $V^{i j} \neq 0$. The corresponding Lagrangian density reads

$$
\mathcal{L}=-\frac{1}{4} F_{\mu \nu} F^{\mu \nu}-\frac{g^{2}}{8} V^{i j} F_{i j} \frac{1}{\nabla^{2}-m^{2}} V^{k l} F_{k l}-A_{0} J^{0}
$$

Following the same steps that led to (16) we find that the potential for two opposite charges located at $\mathbf{y}$ and $\mathbf{y}^{\prime}$ reads ( $\mu$ is a massive cutoff regulator)

$$
V=\frac{e^{2}}{2 \pi} \ln (\mu L) .
$$

Therefore, LIV models with constant spacelike-vectors lead to linear confinement of the test charges while the spacelike case remains coulombic. It is important to mention that a similar study made in $D=4$ models led to analogous results [16].

In summary, in this work we constructed a low energy effective theory that displays Lorentz invariance breaking as the result of condensation of topological defects and studied its physical consequences. The resulting theory exhibits a new extension for the electromagnetic sector of the standard model that is alternative to the extensions proposed so far. This construction uses a condensation mechanism similar to the JTM and describes how a stable effective theory may lead to spontaneous breaking of Lorentz invariance due to the condensation phenomenon, being characterized by constant tensors of any rank. A physical observable related to the existence of this new vacuum was studied which may lead to possible measurable predictions of the Lorentz breaking. As a particular application we have considered the confinement and screening issue for a three-dimensional vector gauge that couples to strings through this LIV-condensation process. The condensation of magnetic strings leads to a massive scalar condensate via this modified JTM. The breaking of Lorentz symmetry is manifest through a constant vector, giving a preferred spacetime direction with distinct consequences. We found that when the condensate constant vector $v_{\mu}$ is purely timelike the resulting static potential remains Coulombic while it was found that when it is purely spacelike the static potential displays a linear confining piece. This result survives any dimensional consideration being valid also in our four-dimensional world. However, in the four-dimensional case we found that the condensation of magnetic string gives rise to a massive vectorial condensate. Since we do not see confined charges, the assumption that Lorentz symmetry is being broken by a constant vector leads us to support 16] a timelike type of vector-breaking as suggested in [11].

This work is partially supported by Fondecyt (Chile) grants 1050546 and 7060050 . C.W. would like to thank $\mathrm{CNPq} / \mathrm{PRONEX} / \mathrm{FAPESQ}$ for financial support.
[1] F. Quevedo and C.A.Trugenberger, Nucl. Phys. B 501, 143 (1997)

[2] V.A.Kostelecky and S.Samuel, Phys.Rev.D39, 683 (1989).

[3] P. Gaete and C. Wotzasek, Phys. Lett. B 601, 108 (2004)

[4] See, V. A. Kostelecky, arXiv:hep-ph/0104227.

[5] D.Colladay and V.A.Kostelecky, Phys.Rev.D 55(1997)6760

[6] See for instance, G.Amelino-Camelia, J.Ellis, N.E.Mavromatos, D.V.Nanopoulos and S.Sarkar, Nature 393(1998)763; J.Alfaro, H.A.Morales-Tecotl and L.F.Urrutia, Phys.Rev.Lett. 84(2000)2318.

[7] G. Amelino-Camelia and T. Piran, Phys.Rev.D64 (2001) 036005; T.Jacobson, S.Liberati, D.Mattingly, Phys.Rev.D66 (2002)081302.

[8] R.Bluhm and V.A.Kostelecky, Phys.Rev.Lett. 84(2000)1381; Phys.Rev.Lett. 84(2000)1101; E. Zavattini et al. [PVLAS Collaboration], Phys.Rev.Lett.96 (2006)110406; B. Feng, M. Li, J. Q. Xia, X. Chen and
X. Zhang, Phys.Rev.Lett.96, 221302(2006)

[9] D.Colladay and V.A.Kostelecky, Phys.Lett.B511 (2001)209; V.A.Kostelecky and R.Lehnert, Phys.Rev.D63 (2001)065008;

[10] For a complet and up to dated list of references see http://www.physics.indiana.edu/ kostelec/

[11] S.M.Carroll, G.B.Field and R.Jackiw, Phys. Rev. D 41, 1231 (1990).

[12] D. Colladay and V. A. Kostelecky, Phys. Rev. D 58, 116002 (1998)

[13] P. Gaete and C. Wotzasek, Phys. Lett. B625, 365 (2005).

[14] P. Gaete, Phys. Rev. D 59, 127702 (1999); P. Gaete and I. Schmidt, Phys. Rev. D 61, 125002 (2000).

[15] P. A. M. Dirac, Can. J. Phys. 33, 650 (1955).

[16] P.Gaete and C.Wotzasek, to appear.

[17] For notational clarity we have dropped out all normalizing factors and signs and adopted a simplified notation proposed in [3]. 